\title{
Detailed Mechanism and Engineering
}

\section{Applicability of Electrolytic Polymerization Aided by a Magnetic Field in Natural Rubber by Mechanical Approach for Sensing (Part 2): Other and Intrinsic Effects on MCF Rubber Property}

\author{
Kunio Shimada ${ }^{*}$, Norihiko Saga ${ }^{2}$ \\ ${ }^{1}$ Department of Symbiotic Systems Sciences, Fukushima University, Fukushima, Japan \\ ${ }^{2}$ Department of Human System Interaction, Kansai Gakuin University, Hyogo, Japan \\ Email: *shimadakun@sss.fukushima-u.ac.jp, saga@kwansei.ac.jp
}

How to cite this paper: Shimada, $K$. and Saga, N. (2016) Detailed Mechanism and Engineering Applicability of Electrolytic Polymerization Aided by a Magnetic Field in Natural Rubber by Mechanical Approach for Sensing (Part 2): Other and Intrinsic Effects on MCF Rubber Property. World Journal of Mechanics, 6, 379-395.

http://dx.doi.org/10.4236/wjm.2016.610027

Received: September 17, 2016

Accepted: October 22, 2016

Published: October 25, 2016

Copyright $\odot 2016$ by authors and Scientific Research Publishing Inc. This work is licensed under the Creative Commons Attribution International License (CC BY 4.0).

http://creativecommons.org/licenses/by/4.0/

\section{Abstract}

The same ordinary electrolytic polymerization of plastic-type polymer solution is applicable to natural rubber, with its $\mathrm{C}=\mathrm{C}$ bonds, if a magnetic field and a filler are added. With the application of a magnetic field and the magnetic responsive fluid known as magnetic compound fluid (MCF), we have clarified the enhancement of the electrolytic polymerization of NR-latex and the growth of the thickness of vulcanized MCF rubber that results from the addition of a magnetic field. The present new method of MCF rubber vulcanization is effective for use in haptic sensors, which are used widely in various engineering applications. In the previous report, part 1 of this study, we investigated many experimental conditions under mechanical approach for sensing: magnetic field strength; applied voltage; electrodes gap; mass concentration, and the ingredients of the MCF. In the present sequential report, part 2, we investigate many other effects on electrolytic polymerization by the same mechanical approach for sensing as in part 1: the Mullins effect; the Piezo effect; vibration; kind of electrode; atmospheric gas. In particular, we clarify that the voltage generates spontaneously in the MCF rubber and that the MCF rubber becomes a Piezo element. These effects on the electrolytic polymerization as well as the effects of the experimental conditions will be useful in engineering applications. By taking the abovementioned parameters and effects into account, MCF rubber that is electrolytically polymerized with the aid of a magnetic field, the use of MCF as a filler, and doping, can be useful in haptic sensor applications. In particular, the effectiveness of the Piezo element can be shown. 


\section{Keywords}

Sensor, Electrolytic Polymerization, Magnetic Field, Magnetic Cluster, Natural Rubber

\section{Introduction}

The development of conductive polymers has become popular, and the principle underlying it is well-known. Since Heeger, MacDiarmid and Shirakawawa were awarded the Nobel Prize in Chemistry in 2000 [1], the method of electrolytic polymerization has been further developed on electrically conductive polymers. The most conductive polymers have $\pi$ bonds such as isoprene $\mathrm{C}=\mathrm{C}$ in the main chain and then become $\pi$-conjugated polymers. The $\pi$-conjugated system is an important factor which produces electrical conductivity. Electrolytic polymerization has been performed using only plastic-type polymer solutions. Rubber is one such polymer solution. There are some rubbers which have $\mathrm{C}=\mathrm{C}$, for example, natural rubber. If natural rubber can be electrically polymerized, it would have electric conductivity.

Shimada has proposed a new method of electrolytic polymerization that involves adding a magnetic field and an MCF as a filler [2] [3]. MCF is a colloidal fluid containing $\mathrm{Fe}_{3} \mathrm{O}_{4}$ and other metal particles on the order of $10 \mathrm{~nm}$ dispersed in a solvent. $\mathrm{Fe}_{3} \mathrm{O}_{4}$ particles have the function of bonding among metal particles, and numerous metal and $\mathrm{Fe}_{3} \mathrm{O}_{4}$ particles are aggregated in the MCF [4]. Therefore, when natural rubber combined with MCF is electrically polymerized, the magnetic cluster is aligned along the direction of the magnetic field lines that are applied in the same direction as the electric field. The polymerized $\mathrm{C}=\mathrm{C}$ arrays along the same direction as the magnetic clusters. At the same time, the $\mathrm{C}=\mathrm{C}$ bonds with each other and is vulcanized by radial polymerization. In addition, the vulcanization reaction to radical polymerization occurs as a result of the carboxyl group $\mathrm{COOH}$ of the oleic acid coating $\mathrm{Fe}_{3} \mathrm{O}_{4}$, and then $\mathrm{C}=\mathrm{C}$ and $\mathrm{Fe}_{3} \mathrm{O}_{4}$ are bonded.

Thus, it is important to focus on and utilize the $\mathrm{C}=\mathrm{C}$ in natural rubber. The magnetic cluster is so-called filler which is commonly used in ordinary plastic-type polymer solution. It can also be said that the polymerization effect is enhanced by utilizing a magnetic field as well as magnetic filler. The rubber electrolytically polymerized by this new method of enhancement of the polymerization that involves adding a magnetic field and an MCF as a filler can be used in engineering applications. The previous report, part 1 of this study, has investigated the effect of the detailed experimental conditions on this new method of electrolytic polymerization: the applied voltage and electric current; the magnetic field strength; the concentration of particles of the MCF; the electrodes gap; the ingredients of MCF rubber, etc. [5]. We clarified the effects of the experimental conditions on the new method by investigating its electric and dynamic characteristics as mechanical approach for sensing. From the effects of magnetic field strength, the electrodes gap, etc. on the electrical characteristics of the MCF rubber as 
shown in part 1 [5], it was clarified that the magnetic cluster has an important role in the mechanism by which electric current flows within MCF rubber. The result can be confirmed by another investigation of the electrical conductivity of MCF rubber liquid under the application of a magnetic field, as shown in Fig. A1 in the Appendix [5], which clarified that the electric current can flow easily through the magnetic clusters. In addition, in the case of the vulcanization of MCF rubber, it was clarified that the effects of the experimental conditions are due to the correlation of the magnetic cluster and the electrolytic polymerization as shown in part 1 of this study [5].

Apart from the experimental conditions, the effects of other parameters in the ordinary electrolytic polymerization of plastic-type conductive polymer solutions, for example, the electrode material, atmospheric gas, and so on, should be investigated. Therefore, in the present sequential report as part 2, we investigated the other effects of parameters at ordinary electrolytic polymerization.

On the other hand, the present new method is distinctive in that it makes use of a magnetic field without the complementary technique of another catalyst or doping in the electrolytic polymerization. If the MCF rubber is doped with any chemicals, elements other than the haptic sensor could be devised [6]-[8], for example, Piezo-electric elements [9], Peltier elements [10], actuating material [11], human electronic skin [12], capacitors, solar batteries [13], organic EL elements [14], and so on, by utilizing ordinary plastic-type polymer solutions. As noted in part 1 of the present report, the same concept used in the electrolytic polymerization of conductive plastic-type solutions could be put to use in the electrolytic polymerization of rubber. Therefore, here in part 2, we conducted an experiment involving doping the MCF rubber and investigating the effect of the doping on the electrical properties of the MCF rubber. As shown in part 1 of this study [5], the experimental condition can be understood to be one of extrinsic effect on the MCF rubber. We can understand that the doping is situated on one of extrinsic effect on the MCF rubber and the intrinsic effect, for example, Piezo effect is occurred by the doping. The intrinsic configuration of the MCF rubber is changed through intermediary electro-chemical reaction by the doping. Mullins effect as well as the Piezo effect can also be situated on intrinsic effect. Because the Mullins effect is relevant to the intrinsic configuration of the MCF rubber. The intrinsic effect as well as the extrinsic effect should be investigated. In part 2, we deal with the Mullins and Piezo effects. Mullins effect is also significant theme at ordinary filled rubber. Because the effect affects the reassurance of rubber's characteristics. On the other hand, the Piezo effect is intriguing subject at the material of rubber. Because the Piezo element is realized by just plastic material. In addition, the effect of doping on the MCF rubber has not been investigated. Therefore, these effects should be clarified in the present study.

\section{Other Effects on Electrolytical Polymerization}

When MCF rubber is utilized in a haptic sensor, more effects must be investigated apart from the parameters investigated in the previous report [5]. The figures shown in this section are the results of using the same experimental procedures used in the previous 
report [3].

In the present report, we also used the experimental apparatus to measure the electric current, the voltage, or the electrical resistance between the electrodes opposing each other upon the application of a normal force to the vulcanized MCF rubber. For convenience of explanation, this experimental procedure is called normal force experimental (NFE). We also used another experimental apparatus to measure the electric current, the voltage, or the electrical resistance within the vulcanized MCF rubber upon the application of a shear force. For convenience of explanation, this experimental procedure is called shear force experimental (SFE).

\subsection{Mullins Effect}

The figures involving NFE previously shown in part 1 [5] give the results when the pressing transverse force was pressed onto the MCF rubber. In general, ordinary rubber filled with any materials undergoes a Mullins effect (stress-softening effect) [15]-[17]. The present MCF rubber is filled rubber. Therefore, we should investigate the Mullins effect which is related to the results involving NFE previously shown in part 1 [5].

The results of repeated stress-strain responses of the tensile MCF rubber are shown in Figure 1. We used a commercial small-size tensile testing machine (SL-6002, IMADA-SS Co. Ltd., Japan) as indicated in the previous report [5]. All test specimens had a thickness of $1 \mathrm{~mm}$, width of $10 \mathrm{~mm}$, and length of $10 \mathrm{~mm}$ at the initial stage before tension was introduced. The maximum tensile force was $0.5 \mathrm{~N}$, and the tensile speed was $100 \mathrm{~mm} / \mathrm{min}$. To contrast with the tensile stress, we investigated the stressstrain responses upon the compression of the MCF rubber. The results of a five-timesrepeated compression and release are shown in Figure 2. The repetition in Figure 2 is irrelevant to that in Figure 1. The arrows in Figure 1 indicate the direction from compression to decompression, and vice versa. In the vulcanization of the MCF rubber, electricity was applied at $6 \mathrm{~V}, 2.7 \mathrm{~A}$, and $188 \mathrm{mT}$, with a 1-mm space between the electrodes. The MCF used consisted of $12 \mathrm{~g}$ of Ni, $3 \mathrm{~g}$ of MF having $40 \mathrm{wt} \% \mathrm{of} \mathrm{Fe}_{3} \mathrm{O}_{4}$ (W-40, Taiho Kozai Co. Ltd., Japan), and NR-latex at $12 \mathrm{~g}$. The duration of the application of

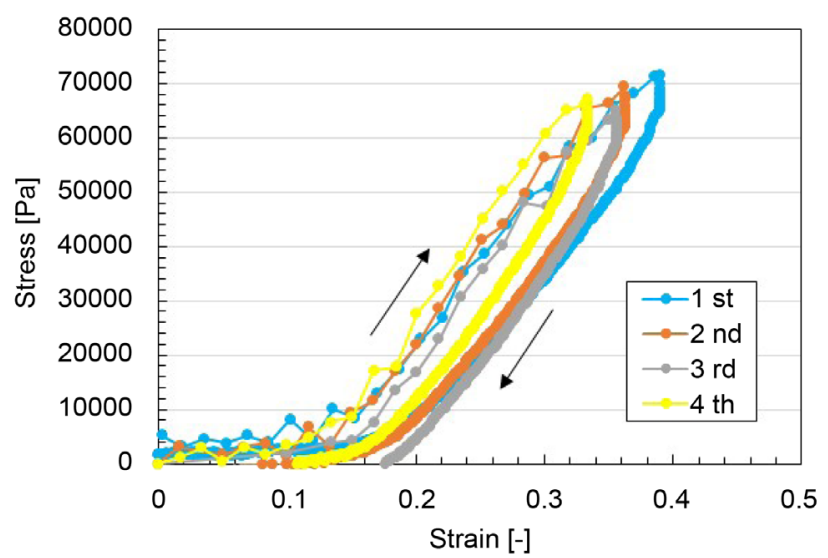

Figure 1. Relation between repeated strain and stress of MCF rubber induced by a tensile testing machine. MCF rubber involves Mullins effect in stress-strain responses. 

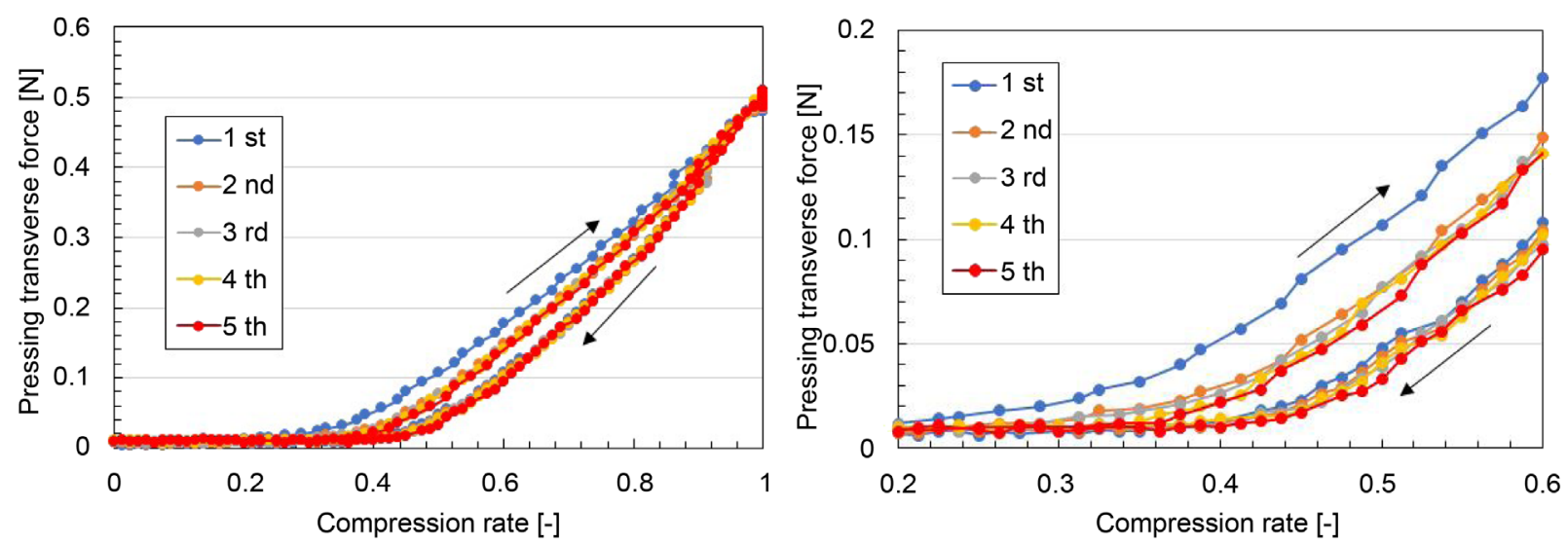

(a)
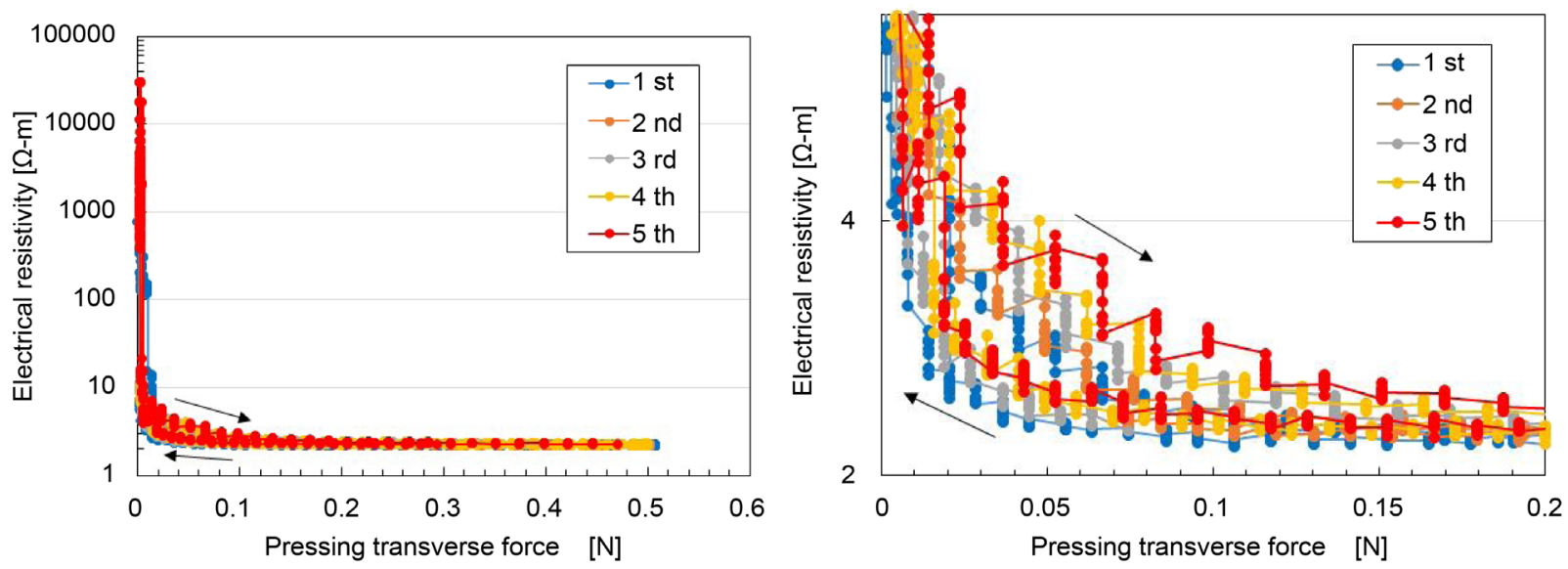

(b)

Figure 2. Compression rate as shown in (a) and electrical resistivity to the repeated pressing transverse force as shown in (b). Respectively, right figures are partially amplified ones of the left figures. MCF rubber involves Mullins effect in NFE responses.

the electric field to the MCF rubber was 30 min. Figure 1 shows the typical result due to the Mullins effect, as shown in other examples [18], such as carbon black-filled rubber [19] [20]. The stress-strain response is non-linear as a result of the filler, as shown in other cases [21].

Figure 2(a) shows the relation between the pressing transverse force and the compression rate. The compression rate at the time of compression is different from that at the release of the force. Therefore, the tendency in the case of compression can be recognized to correspond to that in the tensile MCF rubber as shown in Figure 1. As the case of the stress-strain response is a typical result of the Mullins effect as shown in Figure 1, the case of the pressure-compression response has the Mullins effect as shown in Figure 2(a). Therefore, the electrical resistivity also has a Mullins effect as shown in Figure 2(b). In Figure 2(b), the right at right shows the detailed results of the initial compressing transverse force using NFE. The deviation of the electrical resistivity is within a certain range, and it is comparatively small. We should consider the Mullins effect at the time the present MCF rubber sensor is used. The Mullins effect involves the 
repeatability and the precision of the sensor when measuring electric property under normal force. First, the electric property is different between the application and the removal of the force because of the Mullins effect. As even the ordinary PSECR has also the Mullins effect because of containing filler as shown in Figure A2 in Appendix, its electric property is different between the application and the removal of the force. As for MCF rubber, the measurement error in the present investigation has maximum at $10 \%$ because it changes according to the experimental conditions. It is correspondent to the precision of the MCF sensor. Next, the repeatability has two aspects: duplicability which means the coincidence of the quantitative tendency when another person proceed the measurement at another day; the repetition which means the coincidence of the quantitative tendency when the person proceed the measurement by using the identical specimen multiple times. The former repeatability begets the same quantitative and qualitative tendency such that the MCF rubber is worthy of being used as sensor. The latter repeatability results in the error produced from the changing phenomena and is not different from the precision which results in the error produced from the operation, and has Mullins effect as shown in Figure 2(b).

\subsection{Other Experimental Conditions on Ordinary Conductive Polymers}

The present new method of utilizing electrolytic polymerization in the vulcanization of MCF rubber aided by a magnetic field uses the same method of electrolytic polymerization used in ordinary plastic-type conductive polymer solutions. The latter case is commonly conducted under the atmosphere of an inert gas or Ni gas because of the chemical response of the electrolytically polymerized solution at the electrode surface. Therefore, we investigated the effect of the atmospheric gas on the electrical characteristics. The figures previously shown in part 1 [5] showed the results of experiments conducted under atmospheric conditions. The results under atmospheric conditions, indicated as "Air" in Figure 3, are compared to those under the atmosphere of Ar. On the other hand, electrodes made of Pt are ordinarily used in the electrolytic polymerization of ordinary plastic-type conductive polymer solutions. The electrode material is a very important factor because the electrolytic polymerization is, as it were, electrolysis, and the materials around the anode and cathode undergo electrochemical changes such as electrocatalysis. The electrode material can also provide an element suitable for engineering applications such as haptic sensors and others. The experiments in the previous report [5] were conducted using stainless-made electrodes. In the figures, the stainless-electrodes were marked "stainless". The results of the use of Pt-made electrodes, indicated as "Pt" in Figure 3, were also compared with those using stainless-made electrodes. Figure 3 shows the results for NFE and SFE. The electricity and magnetic field were applied at $6 \mathrm{~V}, 2.7 \mathrm{~A}$, and $188 \mathrm{mT}$, with a 1-mm space between the electrodes. The MCF used consisted of $12 \mathrm{~g}$ of Ni, $3 \mathrm{~g}$ of water-based MF having $40 \mathrm{wt} \%$ of $\mathrm{Fe}_{3} \mathrm{O}_{4}$ (W-40, Taiho Kozai Co. Ltd., Japan), and $12 \mathrm{~g}$ of NR-latex. The duration of the application of the electric field to the MCF rubber was $30 \mathrm{~min}$. The figures in this section show the results obtained using the same experimental procedures of NFE and SFE 


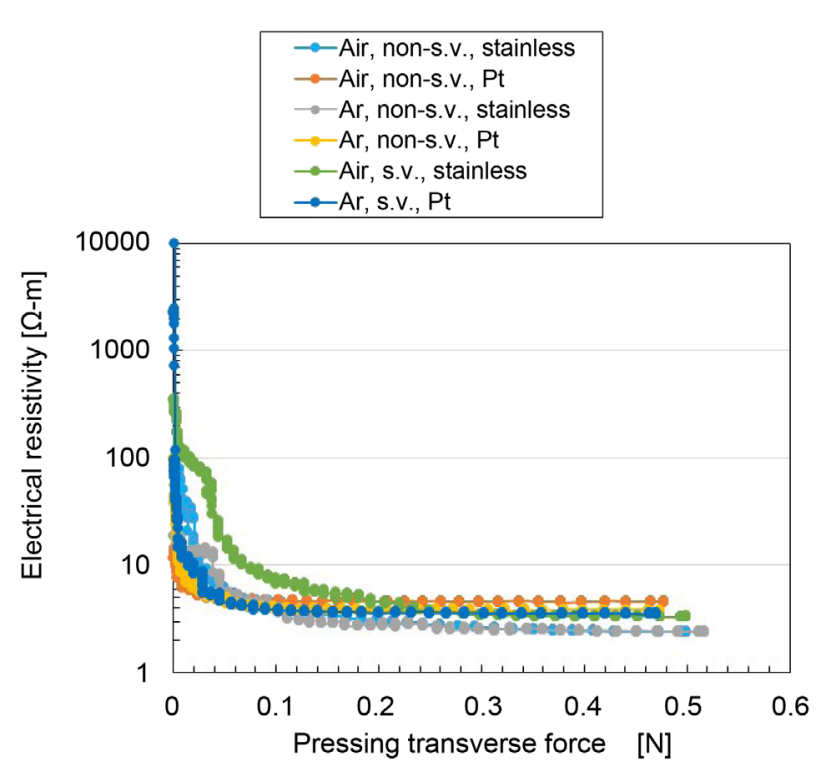

(a)

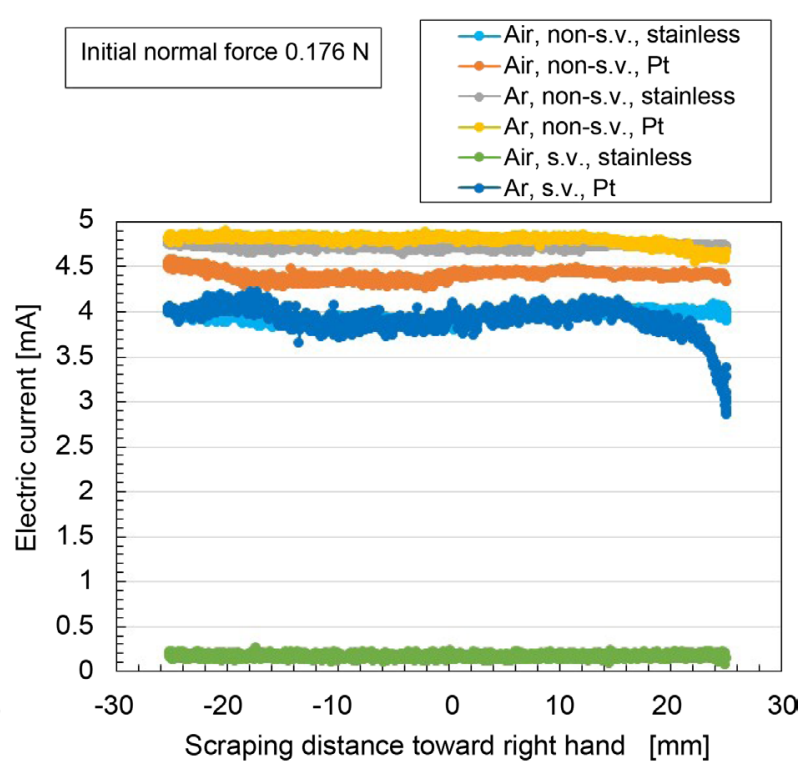

(b)

Figure 3. Effects of the atmospheric gas and the electrode material on the electrical characteristics of the MCF rubber made by the new method: (a) in the case of NFE; (b) in the case of SFE. The vulcanization by the present new method has effect of atmospheric of electrolytic polymerization.

as shown in the last section.

In addition, another report has shown that in the electrolytic polymerization of ordinary plastic-type conductive polymer solutions, the irradiation of supersonic vibration has an effect on the electrolytic synthesis [22]. Therefore, we also investigated the effect of supersonic vibration on the electrical characteristics. In the figure, the effect of the vibration under the application of the electric field is indicated by "s.v.". The container was immersed in water which was immersed in a water bath. The MCF was poured into the container. The supersonic vibration was applied to the container from outside of the water by a supersonic vibrator, i.e. an apparatus containing water, for example, a ultrasonic cleaning machine for glasses. The result without the supersonic vibration is indicated by "non-s.v.".

First, we investigate the results obtained without vibration. With NFE, when we focused on the range of small changes in electrical resistivity under a pressing transverse force of more than 0.1 , we found that no effect of the atmospheric gas occurred with stainless-made electrodes; however, with Pt-made electrodes the effect of the atmospheric gas occurred. The electrical resistivity with Ar was smaller than that with air. On the other hand, with SFE, the electric current with Pt-made electrodes or under an Ar atmosphere was larger than that with stainless-made electrodes or under air. The tendency with NFE was different from that with SFE.

Next, we investigate the effect of the vibration. In both NFE and SFE, the electrical resistivity and electric current were made worse by the vibration. The cause of this can be analyzed based on the appearance within the MCF rubber. Figure 4 shows photographs of cross-sections of MCF rubber both with and without supersonic vibration 


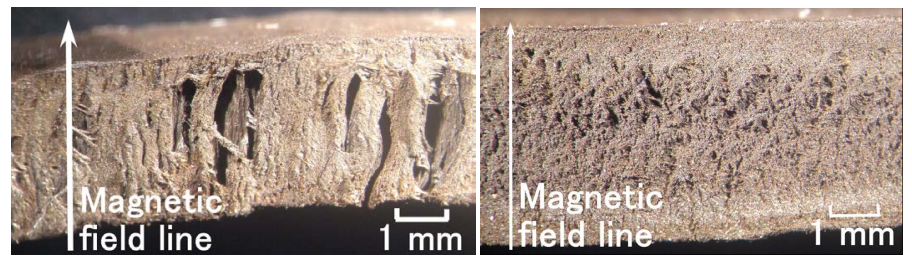

(a)

(b)

Figure 4. Photographs of cross-sections of MCF rubber: A comparison between the case without supersonic vibration (a) and the case with supersonic vibration (b) magnified by microscope. The vulcanization by the present new method has effect of vibration.

magnified by a microscope. The electricity and magnetic field were applied at $6 \mathrm{~V}, 2.7$ $\mathrm{A}$, and $312 \mathrm{mT}$, with a $3-\mathrm{mm}$ space between the electrodes. In order to observe the cross-section clearly, we used an electrodes gap larger than $1 \mathrm{~mm}$. The MCF used consisted of $12 \mathrm{~g}$ of Ni, $3 \mathrm{~g}$ of water-based MF having $50 \mathrm{wt} \%$ of $\mathrm{Fe}_{3} \mathrm{O}_{4}$ (M-300, Sigma Hi-Chemical Co. Ltd., Japan), and $12 \mathrm{~g}$ of NR-latex. The duration of the application of the electric field to the MCF rubber was $30 \mathrm{~min}$. It is clear that the magnetic clusters and $\mathrm{C}=\mathrm{C}$ bonds in the case without vibration formed vertically along the direction of the applied magnetic and electric fields; however, the magnetic clusters and $\mathrm{C}=\mathrm{C}$ bonds were destroyed and dispersed uniformly in the case with the vibration. Nevertheless, the MCF rubber was vulcanized by supersonic polymerization [22]. Therefore, the electric response deteriorated as shown in Figure 3. Thus, the supersonic polymerization leads the electrical properties of the MCF rubber to decrease with the application of the magnetic field in the electrolytic polymerization.

\section{Piezo Effect}

Among ordinary conductive polymers produced by electrolytically polymerizing, some exhibit the Piezo effect. Thus, we investigated the Piezo effect with regard to the MCF rubber produced by the present new method. The figures shown in this section give the results obtained using the same experimental procedures of NFE and SFE used in the previous sections.

First, we deal with the normal force motion. The electricity and the magnetic field were applied at $6 \mathrm{~V}, 2.7 \mathrm{~A}$, and $490 \mathrm{mT}$, with a 3-mm space between the electrodes. The MCF used consisted of $12 \mathrm{~g}$ of Ni, $3 \mathrm{~g}$ of water-based MF having $50 \mathrm{wt} \%$ of $\mathrm{Fe}_{3} \mathrm{O}_{4}$ (M-300, Sigma Hi-Chemical Co. Ltd., Japan), and $12 \mathrm{~g}$ of NR-latex. The duration of the application of the electric field to the MCF rubber was $30 \mathrm{~min}$. Figure 5(a) shows the changes in the induced voltage due to the normal force. The results of four repetitions of the experiment are also shown. In the figure, the arrows show the direction from compression to decompression and vice versa. There exists a Piezo effect in the MCF rubber. Here, this Piezo effect was caused by the use of a 3-mm electrodes gap as follows. In another measurement, it was confirmed that the Piezo effect was very small when we used a 1-mm electrodes gap. We obtained the results of induced voltage ranging from 0.1 to $0.13 \mathrm{mV}$. We found that the induced voltage was influenced by the electrodes gap, and specifically by the thickness of the MCF rubber. As the thickness of 


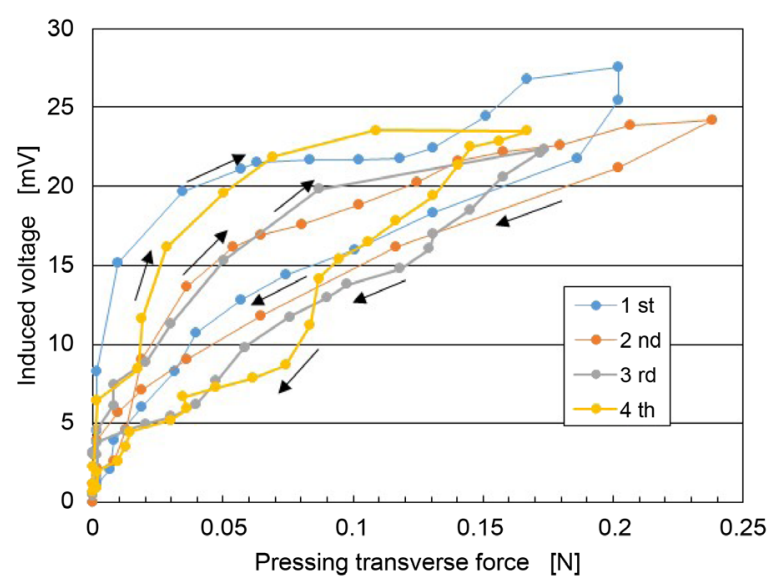

(a)

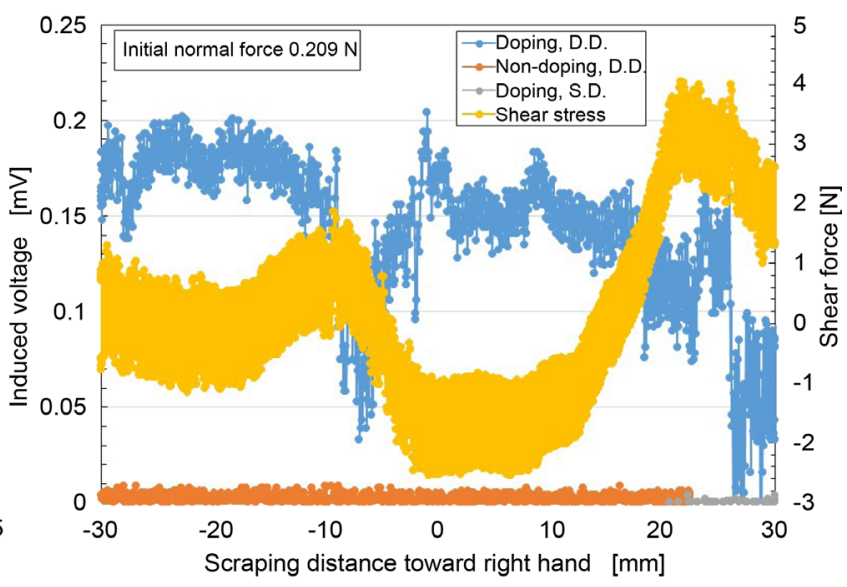

(b)

Figure 5. Piezo effect of the MCF rubber on the electrical characteristics of the MCF rubber made by the new method: (a) in the case of NFE; (b) in the case of SFE.

the MCF rubber became larger, the ionized area within the MCF rubber induced by the electrolytic polymerization became larger, and the induced voltage was enhanced.

On the other hand, the results shown in Figure 5(a) indicate a small changing of the fluctuation of the induced voltage during one path of compression and decompression. Through the 4 repetitions, the changing of the fluctuation is small. This may be related to the fact that the ions within the MCF rubber are formed intricately and have a complicated role, such as a semi-conductor role, and are influenced by the Mullins effect. The NR-latex used contains water, and the MCF rubber contained ions. Between the ions the voltage was induced to occur. The intermingling of the ions within the MCF rubber could evoke the fluctuation of the induced voltage. In order to obtain a stable induced voltage, in Figure 5(a), an insulation paper sheet with a $0.121-\mathrm{mm}$ depth and more than 100-M $\Omega$ resistance was inserted between the MCF rubber and the upper electrode, which is shown in Fig. A4 in the Appendix of the previous report [5]. The surface of the insulation sheet was ionized, and the directions of flow of the ions within the MCF rubber were aligned. Therefore, the magnitude of the fluctuation of the induced voltage decreased. In addition, the small changing of the fluctuation of the induced voltage is reduced by the $\mathrm{C}=\mathrm{C}$ bonds aligned along the magnetic field line created by the electrolytic polymerization, according to a comparison with the results shown in Figure 6. Figure 6 shows the changes in the induced voltage due to the normal force without electrolytic polymerization. The results of five repetitions are also shown. The arrows in the figure function in the same way as those in Figure 5(a). The MCF used consists of $12 \mathrm{~g}$ of Ni, $3 \mathrm{~g}$ of water-based MF having $50 \mathrm{wt} \%$ of $\mathrm{Fe}_{3} \mathrm{O}_{4}$ (M-300, Sigma Hi-Chemical Co. Ltd., Japan), and $12 \mathrm{~g}$ of NR-latex. The MCF rubber was vulcanized by drying under a constant temperature of $47^{\circ} \mathrm{C}$ and the application of a magnetic field at $188 \mathrm{mT}$ without using an electric field. When the electrolytic polymerization was not used, the strength of the $\mathrm{C}=\mathrm{C}$ bonds' alignment along the magnetic field lines was weaker than with electrolytic polymerization. Therefore, through the 4 repetitions, the changing of the fluctuation of the induced voltage is increasing. 


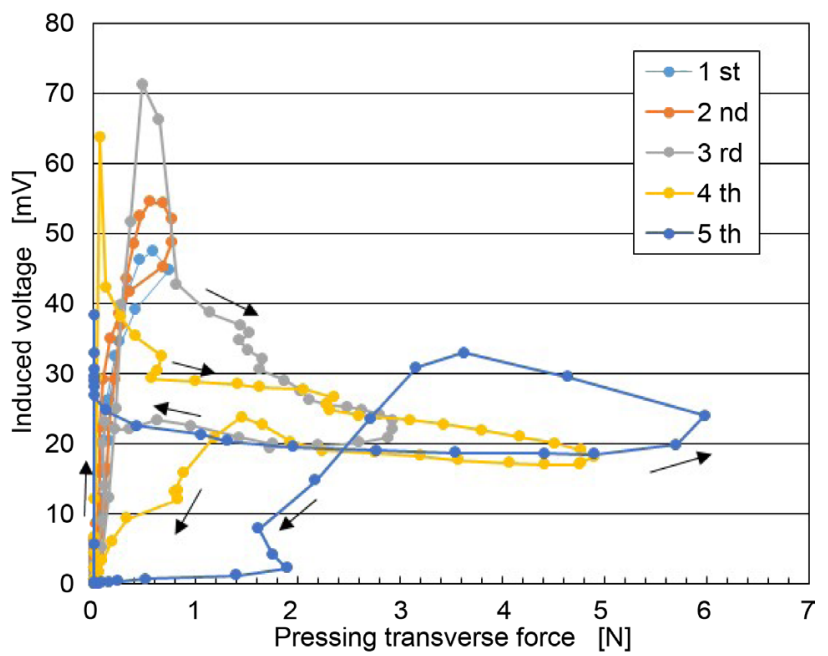

Figure 6. Piezo effect of the MCF rubber on the electrical characteristics of the MCF rubber made using drying only in the case of NFE.

Secondly, we deal with the shear motion. Figure 5(b) shows the changes in the induced voltage in the case of shear motion. The electricity and the magnetic field were applied at $6 \mathrm{~V}, 2.7 \mathrm{~A}$, and $188 \mathrm{mT}$, with a 1- $\mathrm{mm}$ space between the electrodes. The MCF used consisted of $12 \mathrm{~g}$ of Ni, $3 \mathrm{~g}$ of water-based MF having $50 \mathrm{wt} \%$ of $\mathrm{Fe}_{3} \mathrm{O}_{4}$ (M-300, Sigma Hi-Chemical Co. Ltd., Japan), and $12 \mathrm{~g}$ of NR-latex. The duration of the application of the electric field to the MCF rubber was $30 \mathrm{~min}$. Unlike in the case with normal motion in Figure 5(a), no insulation sheet was used. The induced voltage in the case of shear motion was large, even when the MCF rubber thickness was as small as $1 \mathrm{~mm}$, in contrast with the case of normal motion with a 3-mm MCF rubber thickness. The reacting section of the MCF rubber was large, owing to the fact that the electric current flowed between the electrodes longer in the case of shear motion than in the case of normal motion. In addition, in the case of shear motion, the insulation between the MCF rubber and the electrode did not need to be used because the induced voltage was stable.

In the figure, "D.D." and "S.D." signify a difference in the position of the electrodes as shown in Figure A3 in the Appendix. We doped the MCF rubber with a variety of chemical reagents. We used an aqueous potassium iodide solution made up of $60 \mathrm{~g}$ of water and $40 \mathrm{~g}$ of potassium iodide (KI). After the NR-latex, MF, and Ni were mixed using a supersonic vibrator, $4.3 \mathrm{~g}$ of the aqueous KI solution was compounded. The compounded liquid was then mixed again. Here, the choice of MF is important. If W-40 MF (Taiho Kozai Co. Ltd., Japan) is used, in the case of doping with aqueous KI solution, the vulcanized MCF rubber becomes stiff with some cracks and is lacking in elasticity and softness. Therefore, we used M-300 MF. In the figure, the MCF rubber is marked as that with "Doping" and that with "Non-doping".

The induced voltage was very small, as shown in the results indicated "Non-doping, D.D." and "Doping, S.D." These results are clear enough to confirm the large change in the induced voltage in the case of D.D. The doping was effective. The enhancement of 
the induced voltage in the case of D.D. resulted from the fact that the electric current passed through the complicated system of $\mathrm{C}=\mathrm{C}$ bonds, magnetic clusters, ionized molecular, etc., within the vulcanized MCF rubber longer in the case of D.D. than in the case of S.D.. On the other hand, in the figure, the shear stress measured by the load-cell as shown by Fig. A5 in the Appendix of part 1 of this report [5] is also shown. Here, the polarity of the induced voltage is the inverse of that of the shear stress. The qualitative tendency of the induced voltage in the case of "Doping, S.D." in the figure changed according to the changes of the qualitative tendency of the shear stress, and the changing tendency of the shear stress can be obtained quantitatively by the MCF rubber.

In conclusion, the present MCF rubber can be used as a Piezo element in the case of shear motion as well as in the case of normal motion. Furthermore, as the MCF rubber has elasticity and softness, it is useful for applications in which an unbreakable vibrating generator is required.

The photographs of the MCF rubber doped with aqueous KI solution are shown in Figure 7, magnified by microscope. The electricity and the magnetic field were applied at $6 \mathrm{~V}, 2.7 \mathrm{~A}$, and $188 \mathrm{mT}$, with a 2-mm space between the electrodes. The MCF used consists of $12 \mathrm{~g}$ of Ni, $3 \mathrm{~g}$ of water-based MF having $50 \mathrm{wt} \%$ of $\mathrm{Fe}_{3} \mathrm{O}_{4}$ (M-300, Sigma Hi-Chemical Co. Ltd., Japan), and $12 \mathrm{~g}$ of NR-latex. The duration of the application of the electric field to the MCF rubber was $30 \mathrm{~min}$. In Figure 7(c), the upper figure shows the anode side and the lower figure shows the cathode side. Compared to the surfaces of the MCF rubber at the anode and cathode sides without doping, as shown in the previous report [5], the surfaces are rougher due to the electrochemical bond with the chemical reagent of KI as shown in Figure 7(a) and Figure 7(b). The cause of this

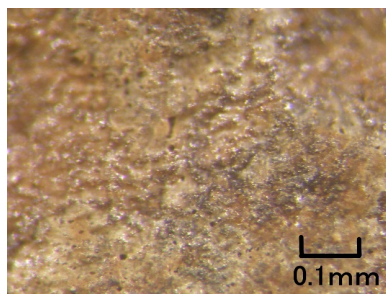

(a)

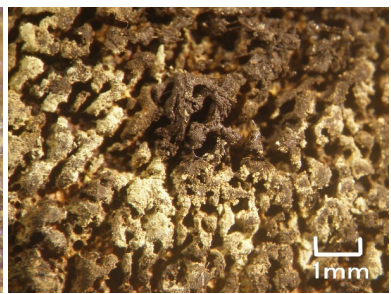

(b)

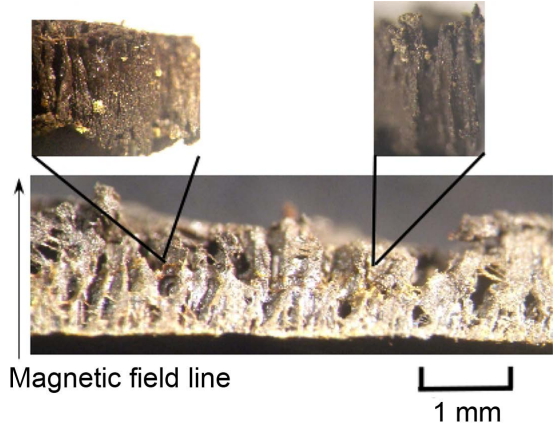

(c)

Figure 7. Photographs of MCF rubber doped with aqueous KI solution magnified by microscope: (a) surface of the MCF rubber anode electrode side; (b) surface of the MCF rubber cathode electrode side; (c) cross-section of the MCF rubber. 
roughness of the surface is the difference between the chemical reaction with doping and that without doping. According to the photographs of cross-sections of the MCF rubber shown in the upper figures in Figure $7(\mathrm{c})$, there was a formation of magnetic clusters and $\mathrm{C}=\mathrm{C}$ bonds aligned with the magnetic field lines, which have the appearance of many stripes that indicate the streak along the magnetic field lines as shown in the magnified images in Figure 7(c). However, there are cavities in places, a result that is different from the cross-sections without doping shown in the previous report [5].

Thirdly, we investigated the stress-strain responses of the tensile MCF rubber with doping. We used the same commercial small-size tensile testing machine as used in Figure 1. The test specimen had a thickness of $1 \mathrm{~mm}$, a width of $10 \mathrm{~mm}$, and a length of $10 \mathrm{~mm}$ at the initial stage before tension. The maximum tensile force was $0.5 \mathrm{~N}$ and the tensile speed was $100 \mathrm{~mm} / \mathrm{min}$. The results of the stress-strain responses of the tensile MCF rubber are shown in Figure 8. In the figure, "with doping" represents the MCF rubber doped with aqueous KI solution as shown in Figure 5, and "without doping" refers to the MCF rubber electrolytically polymerized without doping and consisting of $12 \mathrm{~g}$ of Ni, $3 \mathrm{~g}$ of water-based MF having $40 \mathrm{wt} \%$ of $\mathrm{Fe}_{3} \mathrm{O}_{4}$ (W-40, Taiho Kozai Co. Ltd., Japan), and $12 \mathrm{~g}$ of NR-latex. The electricity and the magnetic field were applied at $6 \mathrm{~V}$, $2.7 \mathrm{~A}$, and $188 \mathrm{mT}$, with a $1-\mathrm{mm}$ space between the electrodes. The duration of the application of the electric field to the MCF rubber was $30 \mathrm{~min}$. The results are from the second tensile stress. It was confirmed that regardless of the differences in the kind of MF, such as M-300 or W-40, the shear-stress response of the tensile MCF rubber shows the same qualitative and quantitative tendencies. Through doping, the MCF rubber becomes soft due to the effect of the doped reagent.

\section{Concluding Remarks}

Focusing on the $\mathrm{C}=\mathrm{C}$ in natural rubber, we conducted electrolytic polymerization aided by the application of a magnetic field and the use of MCF as a filler. We were able to grow the electrolytic polymerization as occurred with plastic in polymer solution. Many enhancements of the electrolytic polymerization investigated by mechanical approach for sensing were indicated as follows.

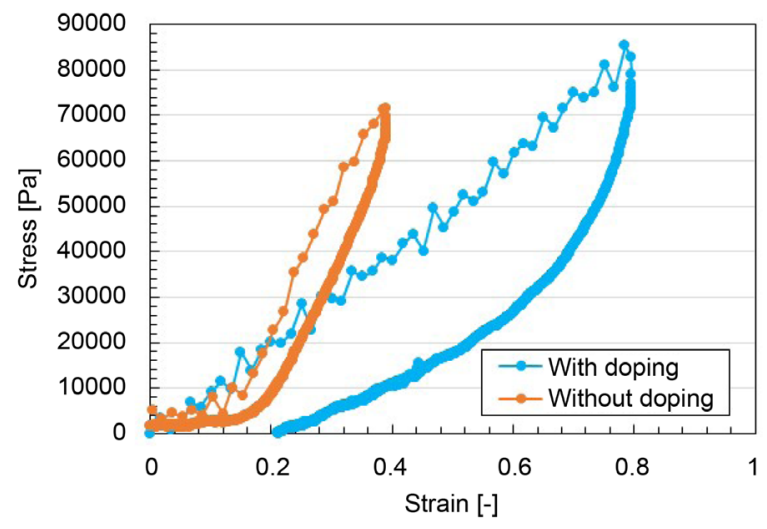

Figure 8. Comparison of strain-stress response of MCF rubber with and without doping using a tensile testing machine. 
The compression rate as the compression force is different than that at the release of the force. The same tendency can be recognized in tensile MCF rubber. This occurs because the electrical resistivity under normal motion also shows a Mullins effect.

Regarding the case with normal motion without vibration, with stainless-made electrodes, no effect of the atmospheric gas was seen; however, with Pt-made electrodes, an effect of the atmospheric gas was observed. The electrical resistivity under an Ar atmosphere was smaller than that under an atmosphere of air. On the other hand, regarding the case involving shear motion, the electric current with Pt-made electrodes or under an Ar atmosphere was larger than that with stainless-made electrodes or air. Thus, the effects of the kinds of electrodes and of the atmosphere with normal motion were different from those with shear motion.

With both normal and shear motion, the electrical properties were made worse by the vibration. This occurred because the magnetic clusters and $\mathrm{C}=\mathrm{C}$ bonds were destroyed by the vibration and were dispersed uniformly.

With compression of the MCF rubber as shown in the case involving normal motion, the voltage generates spontaneously, and a Piezo effect occurs in the MCF rubber. The induced voltage showed a Mullins effect. Regarding the case involving shear motion, the induced voltage became larger due to doping and to the use of cross-passingelectric-current-type electrodes. Doping caused the MCF rubber to become soft due to the effect of the doped reagent.

By taking the above-mentioned parameters and effects into account, MCF rubber that is electrolytically polymerized with the aid of a magnetic field, the use of MCF as a filler, and doping, can be useful in haptic sensor applications. In particular, the effectiveness of the Piezo element can be shown. Furthermore, by doping with other kinds of chemical reagents, other applications can be devised.

\section{Acknowledgements}

A part of writing of this paper was made possible largely through grants from JSPS KAKENHI Grant with Number JP 15K05886, and I would like to acknowledge here the generosity of these organizations.

\section{References}

[1] Shirakawa, H., Louis, E.J., MacDiarmid, A.G., Chiang, C.K. and Heeger, A.J. (1977) Synthesis of Electrically Conducting Organic Polymers: Halogen Derivatives of Polyacetylene, $(\mathrm{CH})_{\mathrm{x}}$. Journal of the Chemical Society, Chemical Communications, 16, 578-580. http://dx.doi.org/10.1039/c39770000578

[2] Shimada, K. (2015) Study of New Making Technique of MCF Haptic Rubber. Proc. JSME Annual Meeting, Mech. Eng. Cong. 2015, Sapporo, 13-16 September 2015, J2220104.

[3] Shimada, K. and Saga, N. (2016) Enhancement of Sensitivity in Natural Rubber Using Electrolytic Polymerization Aided by a Magnetic Field And MCF for Application in Haptic Sensors. Sensors, 16, 1521. http://dx.doi.org/10.3390/s16091521

[4] Shimada, K., Miyazaki, T., Shibayama, A. and Fujita, T. (2003) Extraction of Magnetic Clusters Self-Assembled by a Magnetic Field. Smart Materials and Structures, 12, 297-303. 
http://dx.doi.org/10.1088/0964-1726/12/2/318

[5] Shimada, K. and Saga, N. (2016) Detail Mechanism and Engineering Applicability of Electrolytic Polymerization Aided by Magnetic Field in Natural Rubber: Part 1. The Effect of Experimental Conditions of Electrolytic Polymerization. World Journal of Mechanics, 6, to Be Printed.

[6] Kawasaki, H., Komatsu, T. and Uchiyama, K. (2002) Dexterous Anthropomorphic Robot Hand with Distributed Tactile Sensors: Gifu Hand II. IEEE-ASME Transactions on Mechatronics, 7, 296-303. http://dx.doi.org/10.1109/TMECH.2002.802720

[7] Lee, H.K., Chang, S.I. and Yoon, E. (2009) Dual-Mode Capacitive Proximity Sensor for Robot Application: Implementation of Tactile and Proximity Sensing Capability on a Single Polymer Platform Using Shared Electrodes. IEEE Sensors Journal, 9, 1748-1755.

[8] Kimoto, A., Sugitani, N. and Fujisaki, S. (21010) A Multifunctional Tactile Sensor Based on PVD Films for Identification of Materials. IEEE Sensors Journal, 10, 1508-1513.

[9] Castillo-Castro, T.D., Castillo-Ortega, M.M. and Herrera-Franco, P.J. (2009) Electrical, Mechanical and Piezo-Resistive Behavior of a Polyaniline/Poly(n-butyl methacrylate) Composite. Composites Part A: Applied Science and Manufacturing, 40, 1573-1579. http://dx.doi.org/10.1016/j.compositesa.2009.07.001

[10] McGrail, B.T., Sehirlioglu, A. and Pentzer, E. (2015) Polymer Composites for Thermoelectric Applications. Angewandte Chemie International Edition, 54, 1710-1723.

http://dx.doi.org/10.1002/anie.201408431

[11] Okuzaki, H., Kuwabara, T., Funasaka, K. and Saido, T. (2013) Humidity-Sensitive Polypyrrole Films for Electro-Active Polymer Actuators. Advanced Functional Materials, 23-36, 4400-4407. http://dx.doi.org/10.1002/adfm.201203883

[12] Hammock, M.L., Chortos, A., Tee, B.C.K., Tok, J.B.H. and Bao, Z.A. (2013) 25th Anniversary Article: The Evolution of Electronic Skin (e-skin): A Brief History, Design Considerations, and Recent Progress. Advanced Materials, 25, 5997-6037. http://dx.doi.org/10.1002/adma.201302240

[13] Liu, S.J., Zhang, K., Lu, J.M., Zhang, J., Yip, H.L., Huang, F. and Cao, Y. (2013) High-Efficiency Polymer Solar Cells via the Incorporation of an Amino-Functionalized Conjugated Metallopolymer as a Cathode Interlayer. Journal of the American Chemical Society, 135, 1532615329. http://dx.doi.org/10.1021/ja408363c

[14] Liang, A.H., Zhang, K., Zhang, J., Huang, F., Zhu, X.H. and Cao, Y. (2013) Supramolecular Phosphorescent Polymer Iridium Complexes for High-Efficiency Organic Light-Emitting Diodes. Chemistry of Materials, 25, 1013-1019. http://dx.doi.org/10.1021/cm400333c

[15] Mullins, L. (1969) Softening of Rubber by Deformation. Rubber Chemistry and Technolo$g y$, 42, 339-362. http://dx.doi.org/10.5254/1.3539210

[16] Ogden, R. W. and Roxburgh, D. G. (1999) A Pseudo-Elastic Model for the Mullins Effect in Filled Rubber. Proceedings of the Royal Society A, 455, 2861-2877. http://dx.doi.org/10.1098/rspa.1999.0431

[17] Mars, W. V. (1973) Evaluation of a Pseudo-Elastic Model for the Mullins Effect. Tire Science and Technology, 1, 120-145.

[18] Wang, M.J. (1999) The Role of Filler Networking in Dynamic Properties of Filled Rubber. Rubber Chemistry and Technology, 72, 430-448. http://dx.doi.org/10.5254/1.3538812

[19] Payne, A.R. (1962) The Dynamic Properties of Carbon Black-loaded Natural Rubber Vulcanization. Part I. Journal of Applied Polymer Science, 6, 57-63. http://dx.doi.org/10.1002/app.1962.070061906

[20] Harwood, A.C. and Payne, A.R. (1966) Stress Softening in Natural Rubber Vulcanizates. 
Part III. Carbon Black-Filled Vulcanizates. Journal of Applied Polymer Science, 10, 315 324.

[21] Fletcher, W.P. and Gent, A.N. (1953) Non-Linearity in the Dynamic Properties of Vulcanized Rubber Compounds. Trans. Inst. Rubber Indus., 29, 266-280.

[22] Atobe, M. (2007) Synthesis of Conducting Polymer Materials Having Highly-Regulated Structures in Unique Fields and Media. Chem. Times, 206, 2-9.

[23] Shimada, K. (2015) Experimental Study of Electric Characteristics of Liquid Haptic MCF Sensor Utilizing Biological Natural Rubber. Proc. Jpn. Soc. Fluid Mech. Annual Meeting 2015, Tokyo, 26-28 September 2015, 055. 


\section{Appendix}

Figure A1 shows the electrical conductivity of MCF rubber liquid poured into a container before vulcanization as determined in a previous investigation [23]. The MCF used consists of $12 \mathrm{~g}$ of $\mathrm{Ni}, 3 \mathrm{~g}$ of water-based MF having $50 \mathrm{wt} \%$ of $\mathrm{Fe}_{3} \mathrm{O}_{4}$ (M-300, Sigma Hi-Chemical Co. Ltd., Japan), $16 \mathrm{~g}$ of NR-latex, and water of $31 \mathrm{~g}$. The MCF is diluted more than the MCF used in the present report. However, its mass concentration of the MCF is adequate enough to clarify the effect of the magnetic clusters on its electric property. The electrodes moved from the liquid level toward the bottom of the container, under which a permanent magnet was placed. The magnet caused magnetic clusters to be aggregated on the bottom of the container. The approaching electrodes penetrated into the magnetic clusters. The larger the density of the aggregation became toward the bottom of the container, the more easily the electric current could flow.

Figure A2 shows one of the results of repeated stress-strain responses of PSECR by using the same instrument of a commercial small-size tensile testing machine (SL6002). The test specimens had a thickness of $1 \mathrm{~mm}$, width of $6.2 \mathrm{~mm}$, and length of 10 $\mathrm{mm}$ at the initial stage before tension was introduced. The maximum tensile force was $0.5 \mathrm{~N}$, and the tensile speed was $100 \mathrm{~mm} / \mathrm{min}$. The arrows in Figure A2 indicate the direction from compression to decompression, and vice versa. The figure shows the typical result due to the Mullins effect.

Figure A3 shows a schematic diagram of the positions of "S.D." and "D.D.". S. D. type is the case that the position of both electrodes locates on the same surface of the MCF rubber and D.D. the case that the position of respective electrode locates on the different surface.

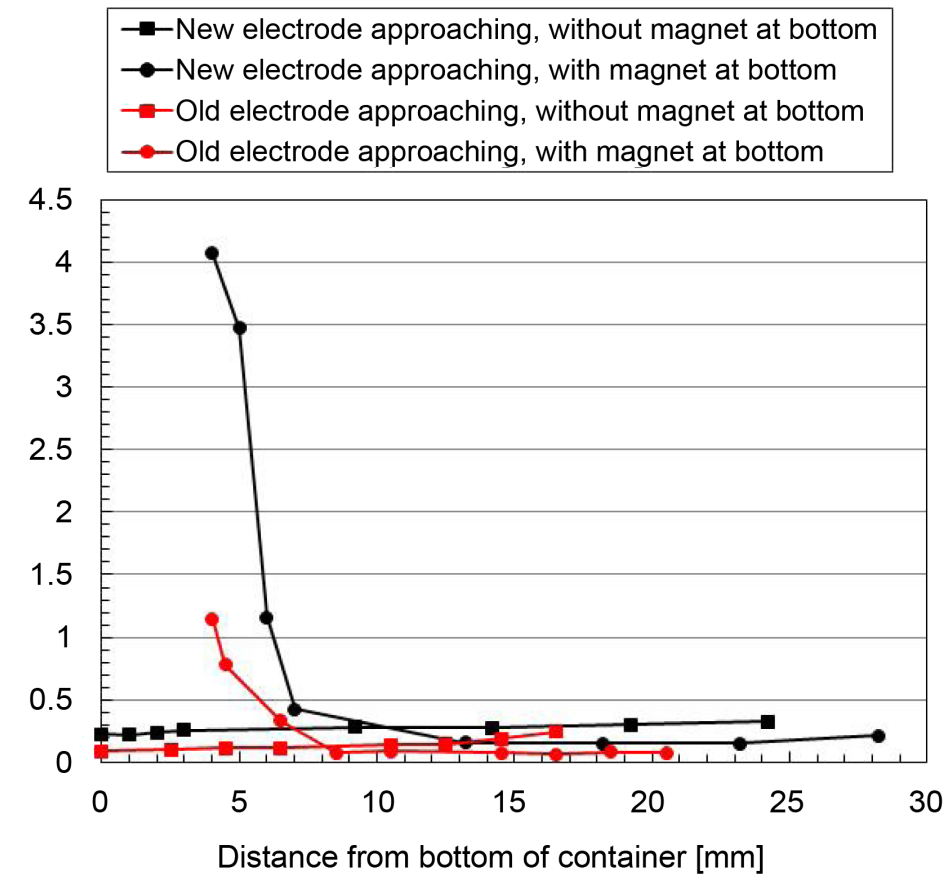

Figure A1. Changing of electrical conductivity as electrodes approached the MCF rubber liquid [23]. 


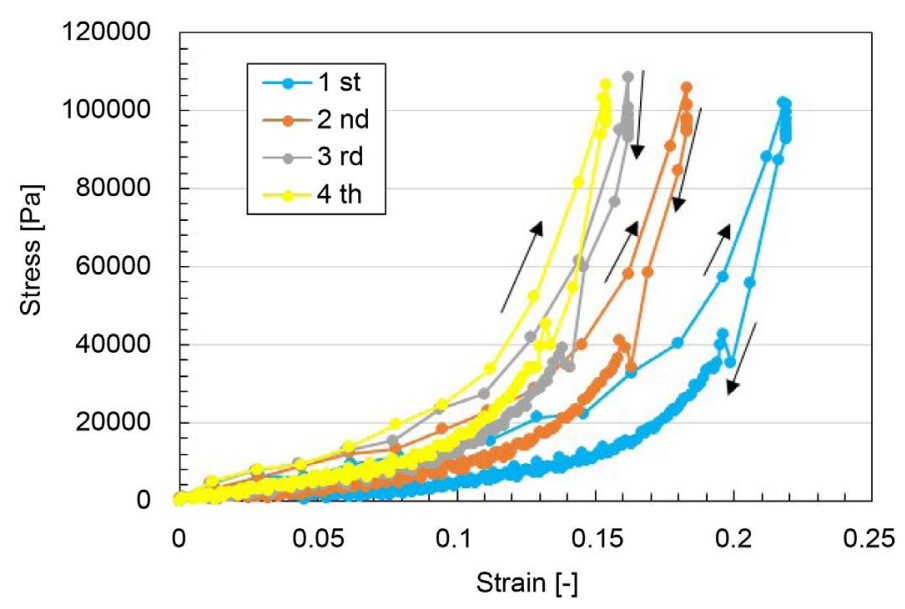

Figure A2. Relation between repeated strain and stress of PSECR induced by a tensile testing machine.

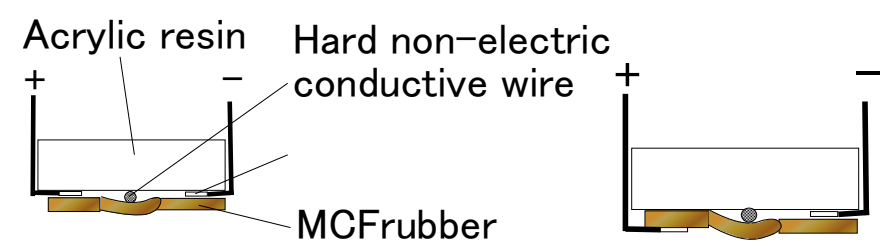

Figure A3. The positions of the electrodes in the case of SFE: the figure on the left shows S.D.; the figure on the right shows D.D.

Submit or recommend next manuscript to SCIRP and we will provide best service for you:

Accepting pre-submission inquiries through Email, Facebook, LinkedIn, Twitter, etc.

A wide selection of journals (inclusive of 9 subjects, more than 200 journals)

Providing 24-hour high-quality service

User-friendly online submission system

Fair and swift peer-review system

Efficient typesetting and proofreading procedure

Display of the result of downloads and visits, as well as the number of cited articles

Maximum dissemination of your research work

Submit your manuscript at: http://papersubmission.scirp.org/

Or contactwjm@scirp.org 